\title{
Supramolecular structure of S-(+)-marmesin-a linear dihydrofuranocoumarin
}

\author{
SANJEEV GOSWAMI, VIVEK K GUPTA*, ASHOK SHARMA ${ }^{\dagger}$ and B D GUPTA ${ }^{\dagger}$ \\ Post-Graduate Department of Physics, University of Jammu, Jammu Tawi 180 006, India \\ ${ }^{\dagger}$ Natural Products Chemistry Division, Regional Research Laboratory, Jammu Tawi 180 001, India
}

MS received 10 June 2005

\begin{abstract}
The title compound, $\mathrm{C}_{14} \mathrm{H}_{14} \mathrm{O}_{4}$, a linear dihydrofuranocoumarin, was isolated from the bark of Aegle marmelos, a plant widely used in Ayurvedic system of medicine for the treatment of various ailments. The crystal structure was determined from $X$-ray diffraction data using direct methods. The compound crystallizes into monoclinic space group $P 2_{1}$ with unit cell parameters: $a=5 \cdot 721(1) \AA, b=13.810(1) \AA$, $c=7 \cdot 864(2) \AA, \beta=100 \cdot 39(1)^{\circ}, Z=2$. The structure was refined by full-matrix least-squares to a final $R$ value of 0.0523 for 1184 observed reflections. The benzopyran moiety is perfectly planar. The dihedral angle between the pyrone and benzene rings is $0 \cdot 3(1)^{\circ}$. The furan ring has a $2 \alpha$-envelope conformation. The molecules are linked by $\mathrm{O}-\mathrm{H}$...O hydrogen bonds into chains and these chains are linked into sheets by $\mathrm{C}-\mathrm{H}$...O hydrogen bonds. Further, the $\pi-\pi$ stacking and $\mathrm{C}-\mathrm{H} . . . \pi$ (arene) interactions link all of the sheets into a supramolecular structure.
\end{abstract}

Keywords. Supramolecular structure; direct methods; hydrogen bond; $\pi-\pi$ interaction; envelope; furanocoumarin.

\section{Introduction}

Furanocoumarins are found to possess dermal photosensitizing activity (Musajo and Rodighiero 1962). The discovery of this unique activity of the furanocoumarins coupled with their utility in the treatment of leucoderma stimulated much activity among researchers seeking to evaluate other naturally occurring furanocoumarins as well as related synthetic compounds for the structureactivity profile of this interesting chemical nucleus. Furanocoumarins have received much attention on account of their ability to perform cycloaddition reactions with DNA during irradiation with UV light (Zarbska 1994; Moor and Gasparro 1996; Brown 2001), a property that has given rise to wide-ranging photochemotherapeutic applications (Miolo et al 1989). These coumarins have also found their way in the treatment of human immunodeficiency disease (North et al 1993). Therefore, these compounds are widely used in the field of medicine, and extensive future research into furanocoumarin derivatives is likely. The present study of the title compound is a part of our program to find the role of non-covalent intermolecular interactions in controlling the crystal packing of

*Author for correspondence (vivek_gupta2k5@yahoo.co.in) CCDC-250695 contains the supplementary crystallographic data for this paper. These data can be obtained free of charge from the Cambridge Crystallographic Data Centre via www.ccdc. cam.ac.uk/data_request/cif. aromatic oxygen heterocycles. The understanding and utilization of all non-covalent interactions is of fundamental importance for further development of supramolecular chemistry and prediction of crystal structures.

\section{Experimental}

S-(+)-marmesin, [2,3-dihydro-2-(1-hydroxy-1-methylethyl)7H-furo[3,2-g][1]benzopyran-7-one], was isolated first by Chatterjee and Mitra (1949) from the bark of Aegle marmelos, a plant commonly known in India as Bael and found wild all over the sub-Himalayan forests. The plant is widely used in Ayurvedic system of medicine for the treatment of various ailments. The bark of the plant has been reported to be used as a remedy in melancholia, intermittent fevers and palpitation of heart (Chopra et al 1956). Marmesin has also been isolated from Ammi majus, a plant used for the treatment of leucoderma (Abumustafa et al 1958). For the present study marmesin was isolated from the stem bark of Aegle marmelos. Dried and powdered stem bark $(1 \mathrm{~kg})$ of Aegle marmelos was extracted with methanol at room temperature and the concentrated extract $(55 \mathrm{~g})$ was chromatographed over a column of silica gel. Elution with ethyl acetate gave S(+)-marmesin, m.p. $189-190^{\circ} \mathrm{C}$. The chemical structure was assigned on the basis of ${ }^{1} \mathrm{H}$ NMR and mass spectral data (figure 1).

Transparent rectangular shaped single crystals of the title compound were grown at room temperature from 


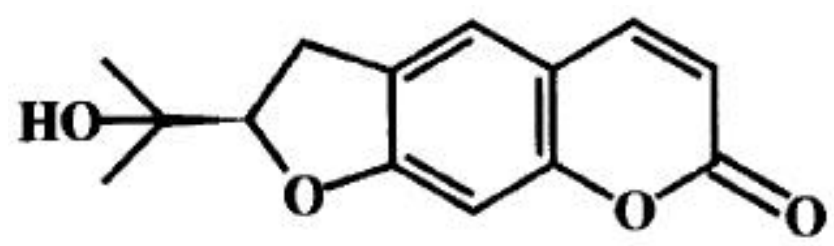

Figure 1. Chemical structure of S-(+)-marmesin.

Table 1. Crystal data and other experimental details.

\begin{tabular}{ll}
\hline CCDC number & 250695 \\
Crystal description & Transparent rectangular \\
Chemical formula & $\mathrm{C}_{14} \mathrm{H}_{14} \mathrm{O}_{4}$ \\
Molecular mass & $246 \cdot 25$ \\
Temperature & $293(2) \mathrm{K}$ \\
Unit cell dimensions & $a=5 \cdot 721(1) \AA, b=13 \cdot 810(1) \AA, c=7 \cdot 864(1) \AA, \beta=100 \cdot 39(1)^{\circ}$ \\
Unit cell volume & $611 \cdot 1(1) \AA^{3}$ \\
$Z$, calculated density & $2,1 \cdot 338 \mathrm{Mgm}^{-3}$ \\
Crystal system, space group & Monoclinic, $P 2_{1}$ \\
$F(000)$ & 260 \\
Radiation/wavelength & CuKo/1.5418 \\
$\theta$ range for entire data collection & $5 \cdot 72<\theta<69 \cdot 9^{\circ}$ \\
Reflections collected/unique & $1200 / 1199[R($ int $)=0 \cdot 0000]$ \\
Observed reflections with $I>2 \sigma(\mathrm{I})$ & 1184 \\
No. of parameters refined & 219 \\
Refinement method & Full-matrix least-squares on $F^{2}$ \\
Goodness-of-fit on $F^{2}$ & $1 \cdot 080$ \\
Final $R$ indices $[I>2 \sigma(\mathrm{I})]$ & $R 1=0 \cdot 0523, w R 2=0 \cdot 1637$ \\
$R$ indices $($ all data) & $R 1=0 \cdot 0526, w R 2=0 \cdot 1646$ \\
$(\Delta / \sigma)_{\text {max }}$ in the final cycle & $-0 \cdot 030($ for $y$ H151) \\
Largest diff. peak and hole & $0 \cdot 246$ and $-0 \cdot 233$ e $\AA^{-3}$ \\
\hline
\end{tabular}

acetone-petroleum ether $(1: 1)$ by slow evaporation technique. Three-dimensional X-ray intensity data were collected on an Enraf Nonius CAD-4 diffractometer with CuK $\alpha$ radiation $(\lambda=1.5418 \AA)$ using a crystal of dimensions $0.4 \times 0.2 \times 0.2 \mathrm{~mm}$. The unit cell dimensions were determined from an angular setting of 25 reflections. The intensity data of 1200 reflections were measured, using the $\omega-2 \theta$ scan technique $(-6 \leq h \leq 6,0 \leq k \leq 16,0 \leq l \leq 9)$. Three standard reflections $(0-22,0-32,1-2$ 2) measured every hour showed no significant variations. The space group was determined to be $P 2_{1}$ from the systematic absences 0k0: $k=2 n+1$. Data were corrected for Lorentz and polarization factors but no absorption correction was made.

The structure was solved by direct methods using SHELXS97 software (Sheldrick 1997). All non-hydrogen atoms of the molecule were obtained from the E-map. Full-matrix least-squares refinement was carried out using SHELXL97 software (Sheldrick 1997). All the hydrogen atoms were located on a difference electron density map and their positional and isotropic thermal parameters
Table 2. Atomic coordinates and equivalent isotropic thermal parameters $\left(\AA^{2}\right)$ for non-hydrogen atoms (e.s.d.'s are given in parenthesis).

\begin{tabular}{lcccc}
\hline Atom & $x$ & $y$ & $z$ & $U_{\text {eq }}{ }^{*}$ \\
\hline O1 & $0 \cdot 9761(4)$ & $0 \cdot 5874(2)$ & $-0 \cdot 1818(3)$ & $0 \cdot 054(1)$ \\
C2 & $1 \cdot 0608(5)$ & $0 \cdot 5612(3)$ & $-0 \cdot 3423(4)$ & $0 \cdot 046(1)$ \\
C3 & $0 \cdot 8749(6)$ & $0 \cdot 4927(3)$ & $-0 \cdot 4405(4)$ & $0 \cdot 049(1)$ \\
C4 & $0 \cdot 5723(5)$ & $0 \cdot 3914(2)$ & $-0 \cdot 2972(4)$ & $0 \cdot 042(1)$ \\
C5 & $0 \cdot 2932(6)$ & $0 \cdot 3108(2)$ & $-0 \cdot 1321(4)$ & $0 \cdot 047(1)$ \\
C6 & $0 \cdot 1990(6)$ & $0 \cdot 3052(3)$ & $0 \cdot 0124(5)$ & $0 \cdot 051(1)$ \\
C7 & $0 \cdot 2792(5)$ & $0 \cdot 3687(2)$ & $0 \cdot 1569(4)$ & $0 \cdot 047(1)$ \\
O7 & $0 \cdot 2125(5)$ & $0 \cdot 3676(2)$ & $0 \cdot 2948(3)$ & $0 \cdot 061(1)$ \\
O8 & $0 \cdot 4470(4)$ & $0 \cdot 4367(2)$ & $0 \cdot 1379(3)$ & $0 \cdot 051(1)$ \\
C9 & $0 \cdot 7130(6)$ & $5 \cdot 0157(3)$ & $-0 \cdot 0104(4)$ & $0 \cdot 049(1)$ \\
C10 & $0 \cdot 8096(5)$ & $0 \cdot 5220(2)$ & $-0 \cdot 1592(4)$ & $0 \cdot 042(1)$ \\
C11 & $0 \cdot 7417(5)$ & $0 \cdot 4612(2)$ & $-0 \cdot 3022(4)$ & $0 \cdot 042(1)$ \\
C12 & $0 \cdot 4703(5)$ & $0 \cdot 3809(2)$ & $-0 \cdot 1479(4)$ & $0 \cdot 039(1)$ \\
C13 & $0 \cdot 5435(5)$ & $0 \cdot 4433(2)$ & $-0 \cdot 0095(4)$ & $0 \cdot 040(1)$ \\
C14 & $1 \cdot 1077(5)$ & $0 \cdot 6541(2)$ & $-0 \cdot 4345(3)$ & $0 \cdot 041(1)$ \\
C15 & $1 \cdot 2008(7)$ & $0 \cdot 6276(3)$ & $-0 \cdot 5990(5)$ & $0 \cdot 059(1)$ \\
C16 & $1 \cdot 2853(6)$ & $0 \cdot 7172(3)$ & $-0 \cdot 3178(5)$ & $0 \cdot 057(1)$ \\
O17 & $0 \cdot 8886(4)$ & $0 \cdot 7038(2)$ & $-0 \cdot 4899(3)$ & $0 \cdot 054(1)$ \\
\hline
\end{tabular}

$* U_{\mathrm{eq}}=(1 / 3) \Sigma_{i} \Sigma_{j} \mathrm{U}_{i j} a_{i}^{*} a_{j}^{*} a_{i} \cdot a_{j}$ 
Table 3. Selected bond lengths $(\AA)$ and bond angles $\left({ }^{\circ}\right)$ for non hydrogen atoms (e.s.d.'s are given in parentheses).

\begin{tabular}{lrlc}
\hline C5-C6 & $1 \cdot 345(4)$ & C5-C12 & $1 \cdot 423(4)$ \\
C6-C7 & $1 \cdot 443(5)$ & C7-O7 & $1 \cdot 214(4)$ \\
C7-O8 & $1 \cdot 370(4)$ & O8-C13 & $1 \cdot 374(3)$ \\
C6-C7-O7 & $126 \cdot 1(3)$ & O7-C7-O8 & $116 \cdot 5(3)$ \\
C6-C7-O8 & $117 \cdot 4(3)$ & C4-C12-C5 & $123 \cdot 5(3)$ \\
O8-C13-C9 & $116 \cdot 0(2)$ & - & - \\
O1-C2-C3-C11 & $-17 \cdot 4(3)$ & C2-C3-C11-C10 & $12 \cdot 3(3)$ \\
C3-C11-C10-O1 & $-2 \cdot 2(4)$ & C11-C10-O1-C2 & $-9 \cdot 5(3)$ \\
C10-O1-C2-C3 & $17 \cdot 1(3)$ & & - \\
\hline
\end{tabular}

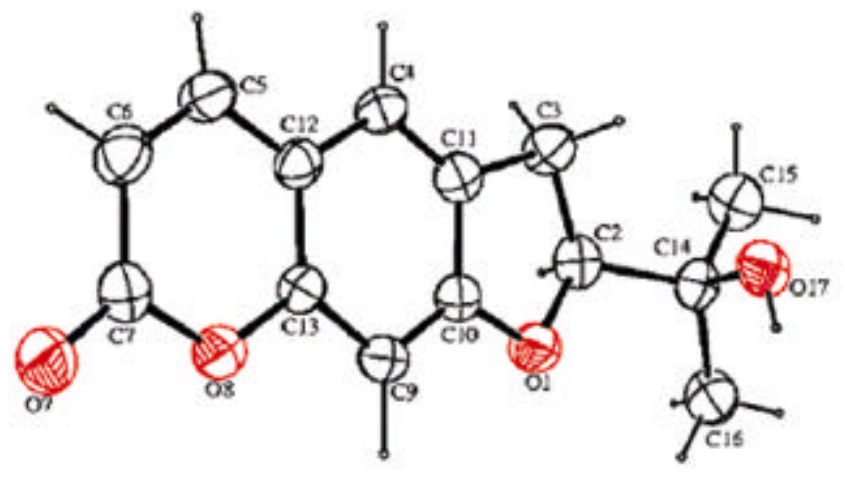

Figure 2. ORTEP view of the molecule with the atomlabelling scheme. Displacement ellipsoids are drawn at the $50 \%$ probability level and $\mathrm{H}$ atoms are shown as small spheres of arbitrary radii.

were included in the refinement. The final refinement cycles converged were $R=0.0523$ and $w R\left(F^{2}\right)=0 \cdot 1637$. The final weighting scheme was $w=1 /\left[\sigma^{2}\left(F_{0}^{2}\right)+(0 \cdot 1552 P)^{2}+\right.$ $0 \cdot 02 P]$, where $P=\left[F_{0}^{2}+2 F_{\mathrm{c}}^{2}\right] / 3$. Maximum shift to e.s.d. ratio for all atoms in the final cycle was -0.030 (for $y$, H151). Atomic scattering factors were taken from International tables for X-ray Crystallography (1992, Vol. C, tables $4 \cdot 2 \cdot 6 \cdot 8$ and $6 \cdot 1 \cdot 1 \cdot 4)$. The crystallographic data are summarized in table 1. CCDC-250695 contains the supplementary crystallographic data for this paper.

\section{Results and discussion}

The final atomic coordinates with equivalent isotropic displacement parameters are presented in table 2. Selected bond distances, bond angles and torsion angles are listed in table 3. An ORTEP view of the title compound with atomic labeling is shown in figure 2 (Farrugia 1997). The geometry of the molecule has been calculated using the software winGX (Farrugia 1999) and PARST (Nardelli 1983, 1995).

The bond distances and bond angles of the title compound are in good agreement with the corresponding values reported for related furanocoumarin derivatives (Stemple and Watson 1972; Gupta et al 1993; Singh et al

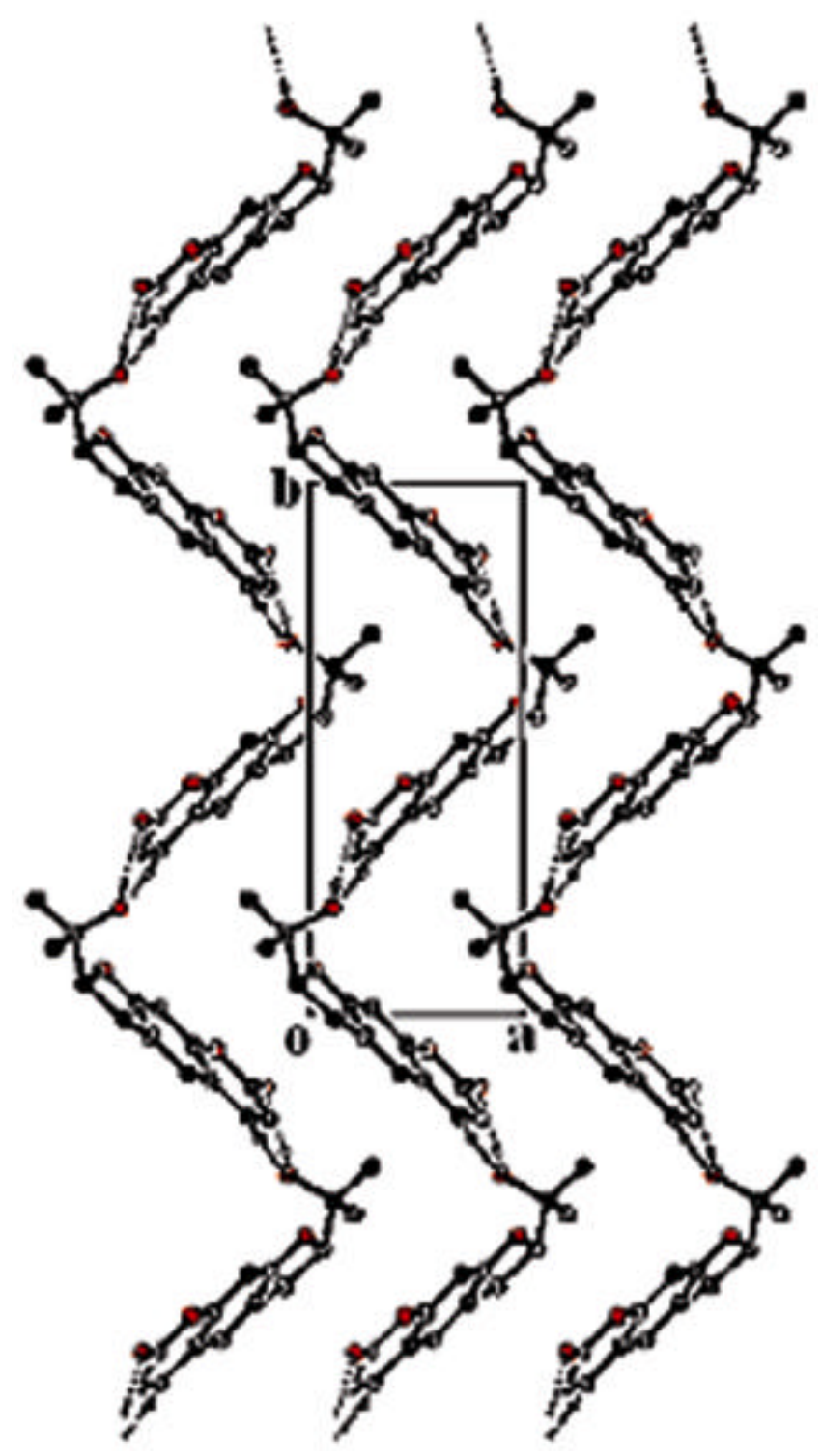

Figure 3. Part of the crystal structure showing the formation of $\mathrm{O}-\mathrm{H} . . . \mathrm{O}$ chains along $b$-axis.

1995; Magotra et al 1995). In the benzopyran ring, the

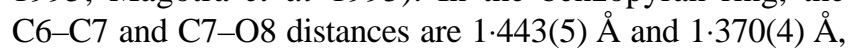
respectively, indicating that the electrons are delocalized 


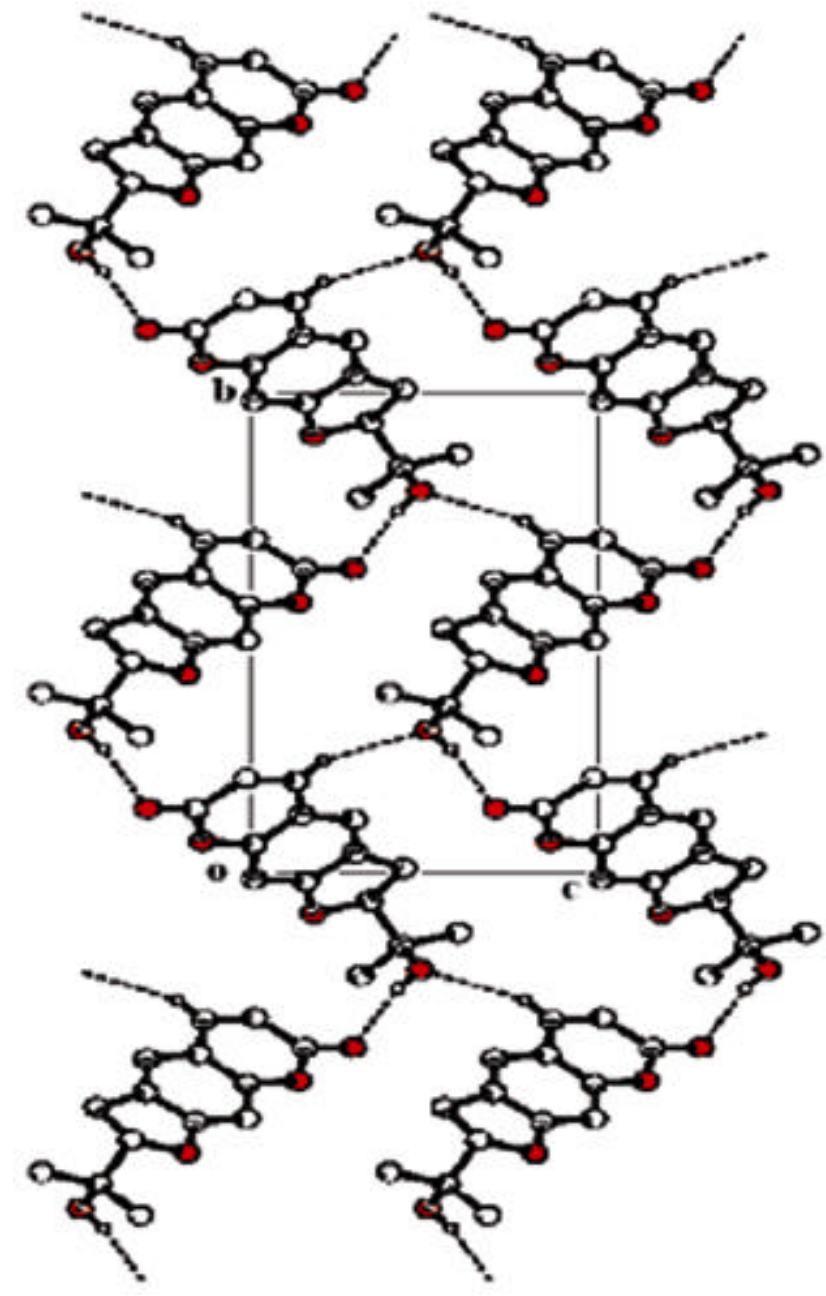

Figure 4. Part of the crystal structure down $a$-axis, showing the linking of chains into sheets by $\mathrm{C}-\mathrm{H}$...O hydrogen bond. For clarity, only $\mathrm{H}$ atoms involved in hydrogen bonding have been included.

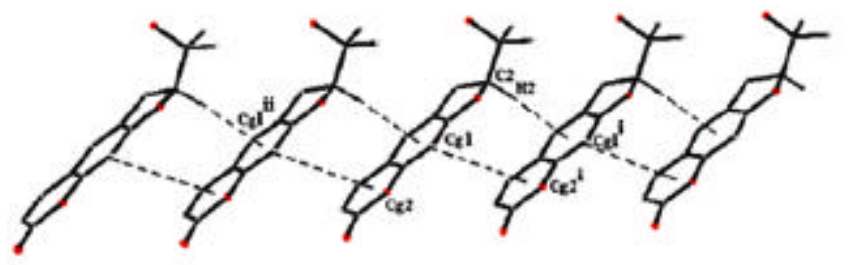

Figure 5. Molecular stacking along the $a$-axis showing the linking of the molecules by $\mathrm{C}-\mathrm{H} . . . \pi$ and $\pi-\pi$ interactions. Ring centroids involved in the $\mathrm{C}-\mathrm{H} \ldots \pi$ and $\pi-\pi$ interactions are joined by dashed lines.

in the ring with the carbonyl group acting as the electronwithdrawing group. This is corroborated by the fact that the benzopyran ring is planar (maximum deviation, 0.014(4) $\AA$ for C6). The angles O8-C13-C9 and C4-C12-C5 at the junction of the pyrone and benzene rings are, respec- tively, smaller and greater than $120^{\circ}$. This causes the approach of $\mathrm{O} 8$ to $\mathrm{C} 9$ and the separation of $\mathrm{C} 4$ from $\mathrm{C} 5$. This feature found in coumarin derivatives is also present in the title compound. The widening of the angle O7-C7$\mathrm{O} 8$ is another commonly occurring feature which is usually observed in coumarin systems and the large value of this angle is attributed to the lone-pair interactions between $\mathrm{O} 7$ and $\mathrm{O} 8$.

The furan ring has a $2 \alpha$-envelope conformation with a phase angle of pseudorotation, $\Delta=25.47^{\circ}$ and maximum angle of torsion, $\phi_{\mathrm{m}}=17.84^{\circ}$ (Altona et al 1968). The asymmetry parameter, $\Delta \mathrm{C}_{\mathrm{s}}(\mathrm{C} 2)$, which gives the distortion from ideal mirror symmetry bisecting the C10-C11 bond is 1.99 (Duax and Norton 1975). Atom C2 is disposed $-0 \cdot 275(3) \AA$ below the plane defined by the other four ring atoms. The dihedral angle between the planes of furan ring and benzopyran moiety is $3 \cdot 8(1)^{\circ}$.

The supramolecular structure of marmesin is dictated by two intermolecular hydrogen bonds. The stronger of these two hydrogen bonds (O17-H17O...O7) gives rise to a chain running parallel to the [010] direction. Atom $\mathrm{O} 17$ of the hydroxy group in the molecule at $(x, y, z)$ acts as a hydrogen-bond donor to carbonyl atom $\mathrm{O} 7$ in the molecule at $(-x+1, y+1 / 2,-z)$, producing a chain (figure $3)$. The chains are linked into sheets by the second hydrogen bond (C5-H5...O17) (figure 4). Atom C5 in the molecule at $(x, y, z)$ acts as a hydrogen bond donor to hydroxy atom $\mathrm{O} 17$ in the molecule at $(-x+1, y-1 / 2$, $-z+1)$. There is one $\mathrm{C}-\mathrm{H} . . . \pi$ (arene) hydrogen bond with H...centroid distance of less than $3.0 \AA$ which serves to link all of the sheets into a single three-dimensional framework. Atom $\mathrm{C} 2$ in the molecule at $(x, y, z)$ acts as a hydrogen-bond donor to the benzene ring in the molecule at $(x+1, y, z)$. The three-dimensional framework of marmesin is further stabilized by $\pi-\pi$ interactions between the pyrone and phenyl rings (figure 5). The interacting molecules are related by unit-cell translations along the short $a$-axis. Details of C-H... $\pi, \mathrm{C}-\mathrm{H} \ldots \mathrm{O}, \mathrm{O}-$ $\mathrm{H}$... O hydrogen bonds, and $\pi-\pi$ stacking interactions are given in tables 3 and 4 , respectively.

\section{Conclusions}

(I) The dihedral angle between the pyrone and benzene rings is only $0 \cdot 3(1)^{\circ}$, indicating the perfect planarity of the benzopyran moiety. The planarity of the benzopyran moiety confirms the aromatic character of this system.

(II) It is found in coumarin derivatives that the angles at the junction of the pyrone and benzene rings deviate significantly from $120^{\circ}$. This feature is also present in the title compound.

(III) An analysis of $\pi-\pi$ interactions reveals that $\pi-\pi$ stacking is an offset arrangement of the rings.

(IV) C-H..., , C-H...O, O-H...O hydrogen bonds, and $\pi-\pi$ stacking interactions play a crucial part in assem- 
Table 4. $\mathrm{C}-\mathrm{H} \ldots \pi, \mathrm{C}-\mathrm{H} \ldots \mathrm{O}$ and $\mathrm{O}-\mathrm{H} . . . \mathrm{O}$ hydrogen bonding geometry. $\mathrm{Cg} 1$ represents the centre of gravity of the phenyl ring.

\begin{tabular}{|c|c|c|c|c|}
\hline D-H...A & D-H $(\AA)$ & D...A $(\AA)$ & H...A $(\AA)$ & $\mathrm{D}-\mathrm{H} \ldots \mathrm{A}\left({ }^{\circ}\right)$ \\
\hline $\mathrm{C} 2-\mathrm{H} 2 \ldots \mathrm{Cg} 1^{\mathrm{i}}$ & $0.99(3)$ & $3 \cdot 703$ & $2 \cdot 721$ & 169 \\
\hline $\mathrm{C} 3-\mathrm{H} 32 \ldots \mathrm{O} 17$ & $1 \cdot 04(6)$ & $2 \cdot 944(5)$ & $2 \cdot 45(7)$ & $108(4)$ \\
\hline C5-H5 ..O $17^{\mathrm{iii}}$ & $0 \cdot 86(6)$ & $3 \cdot 314(4)$ & $2 \cdot 51(5)$ & $156(4)$ \\
\hline $\mathrm{O} 17-\mathrm{H} 17 \mathrm{O} \ldots \mathrm{O} 7^{\mathrm{iv}}$ & $0 \cdot 78(6)$ & $2 \cdot 850(4)$ & $2 \cdot 09(6)$ & $167(6)$ \\
\hline
\end{tabular}

Symmetry code: (i) $x+1, y, z$; (iii) $-x+1, y-1 / 2,-z+1$; (iv) $-x+1, y+1 / 2,-z$

Table 5. Geometry of $\pi-\pi$ interactions. Cg represents the centre of gravity of the following rings: $\mathrm{Cg} 1$ phenyl ring, $\mathrm{Cg} 2$ pyrone ring. $\mathrm{CgI} . . \mathrm{CgJ}$ represents the distance between the ring centroids; $\mathrm{CgI}$...P, the perpendicular distance of the centroid of one ring from the plane of the other. $\alpha$ is the dihedral angle between the planes of rings I and $\mathrm{J} ; \beta$ is the angle between normal to the centroid of ring $\mathrm{I}$ and the line joining ring centroids; $\Delta$ is the displacement of the centroid of ring $J$ relative to the intersection point of the normal to the centroid of ring I and the least-squares plane of ring $\mathrm{J}$.

\begin{tabular}{lcccccc}
\hline $\mathrm{CgI}$ & $\mathrm{CgJ}$ & $\mathrm{CgI} \ldots \mathrm{CgJ}(\AA)$ & $\mathrm{CgI} \ldots \mathrm{P}(\AA)$ & $\alpha\left(^{\circ}\right)$ & $\beta\left({ }^{\circ}\right)$ & $\Delta(\AA)$ \\
\hline 1 & $2^{\mathrm{i}}$ & 4.276 & 3.775 & 0.36 & $28 \cdot 37$ & $2 \cdot 00$ \\
2 & $1^{\mathrm{ii}}$ & 4.276 & 3.763 & 0.36 & $28 \cdot 01$ & $2 \cdot 03$ \\
\hline
\end{tabular}

Symmetry code: (i) $x+1, y, z$ and (ii) $x-1, y, z$

bling the molecules into an organized supramolecular structure.

\section{Acknowledgements}

The authors are grateful to Prof. T P Singh, Department of Biophysics, All India Institute of Medical Sciences, New Delhi, for providing the intensity data collection facility. (VKG) is grateful to the University Grants Commission, Govt. of India, for research funding under DRS Program [Project No. F-530/1/DRS/2001(SAP-I)].

\section{References}

Abumustafa E A, Badran N, Fayez M B E and Starkowsky N A 1958 Nature 15482

Altona C, Geise H J and Romers C 1968 Tetrahedron 2413

Brown D A 2001 J. Photochem. Photobiol. B63 148

Chatterjee A and Mitra S S 1949 J. Am. Chem. Soc. 71606

Chopra R N, Nayar S L and Chopra I C 1956 Glossary of Indian medicinal plants (New Delhi: CSIR) p. 8
Duax W and Norton D A 1975 Atlas of steroid structures (New York: Plenum) p. 1

Farrugia L J 1997 J. Appl. Cryst. 30565

Farrugia L J 1999 J. Appl. Cryst. 32837

Gupta V K, Rajnikant, Goswami K N, Mazumdar S K, Gupta B D and Banerjee S K 1993 Cryst. Res. Technol. 28187

Magotra D K, Gupta V K, Rajnikant, Goswami K N and Gupta B D 1995 Acta Crystallogr. C51 2637

Miolo G, Stefanids M, Santella R M, Dall'Acqua F and Gasparro F P 1989 J. Photochem. Photobiol. B3 102

Moor A C E and Gasparro F P 1996 Clin. Dermatol. 14353

Musajo L and Rodighiero G 1962 Experientia 18153

Nardelli M 1983 Comput. Chem. 795

Nardelli M 1995 J. Appl. Cryst. 28659

North J, Neyndorff H and Levy J 1993 J. Photochem. Photobiol. B17 99

Sheldrick G M 1997 SHELXS97 and SHELXL97 (Germany: University of Gottingen)

Singh A, Gupta V K, Rajnikant and Goswami K N 1995 Mol. Mater. 5289

Stemple N R and Watson W H 1972 Acta Crystallogr. B28 2485

Zarbska Z 1994 J. Photochem. Photobiol. B23 101 